\title{
Myocardial Fat Imaging
}

\author{
Peter Kellman • Diego Hernando • Andrew E. Arai
}

Published online: 11 March 2010

(C) The Author(s) 2010. This article is published with open access at Springerlink.com

\begin{abstract}
The presence of intramyocardial fat may form a substrate for arrhythmias, and fibrofatty infiltration of the myocardium has been shown to be associated with sudden death. Therefore, noninvasive detection could have high prognostic value. Fat-water-separated imaging in the heart by MRI is a sensitive means of detecting intramyocardial fat and characterizing fibrofatty infiltration. It is also useful in characterizing fatty tumors and delineating epicardial and/or pericardial fat. Multi-echo methods for fat and water separation provide a sensitive means of detecting small concentrations of fat with positive contrast and have a number of advantages over conventional chemical-shift fat suppression. Furthermore, fat and water-separated imaging is useful in resolving artifacts that may arise due to the presence of fat. Examples of fat-water-separated imaging of the heart are presented for patients with ischemic and nonischemic cardiomyopathies, as well as general tissue classification.
\end{abstract}

P. Kellman $(\bowtie)$

Laboratory of Cardiac Energetics,

National Institutes of Health/NHLBI,

10 Center Drive, msc-1061,

Bethesda, MD 20892-1061, USA

e-mail: kellmanp@nhlbi.nih.gov

D. Hernando

Beckman Institute for Advanced Science and Technology,

University of Illinois at Urbana-Champaign,

Urbana, IL, USA

e-mail: dhernan2@illinois.edu

\section{A. E. Arai}

Laboratory of Cardiac Energetics,

National Institutes of Health/NHLBI,

10 Center Drive, msc-1061,

Bethesda, MD 20892-1061, USA

e-mail: araia@nhlbi.nih.gov
Keywords Cardiac MR - Intramyocardial fat · Fibrofatty . Chronic MI - Late enhancement - Lipoma

\section{Introduction}

Fatty infiltration of the myocardium may appear in isolation or in association with fibrous tissue [1-3]. Intramyocardial fat is correlated with various cardiomyopathies but is also present to some degree in normal myocardium. The pathophysiology of fibrofatty infiltration is not fully understood. Fatty infiltration of fibrous tissue is observed in imaging studies done by both CT [4-7] and MRI [8-11••, 12], and has been reported in a number of histology studies of samples from endocardial biopsies as well as autopsies [13-16]. The mechanism of fatty infiltration, referred to as lipomatous metaplasia, has been described by several authors [15]. The measurement of fibrofatty infiltration by imaging studies is relatively new. As a result, the prevalence and factors that influence lipomatous metaplasia are largely unknown. The clinical implications are also not established, although this is a subject of active research.

Histological evidence of fibrofatty infiltration is a hallmark of arrhythmogenic right ventricular dysplasia (ARVD), and is also evident in chronic myocardial infarction (MI) and other nonischemic cardiomyopathies. The presence of intramyocardial fat may form a substrate for arrhythmias [17, 18] due to the lower electrical conductivity of fat. It has been shown that fibrofatty infiltration of the myocardium is associated with sudden death [1], and therefore noninvasive detection could have high prognostic value. Fat-waterseparated imaging in the heart by MRI $[11 \bullet, 12,19 \bullet \cdot]$ is a sensitive means of detecting intramyocardial fat and characterizing fibrofatty infiltration. It is also useful in characterizing fatty tumors and delineating epicardial and/or pericardial fat, and in distinguishing pericardial disease.

In addition to the presence of adipocytes that infiltrate the myocardium, there is growing interest in the mea- 


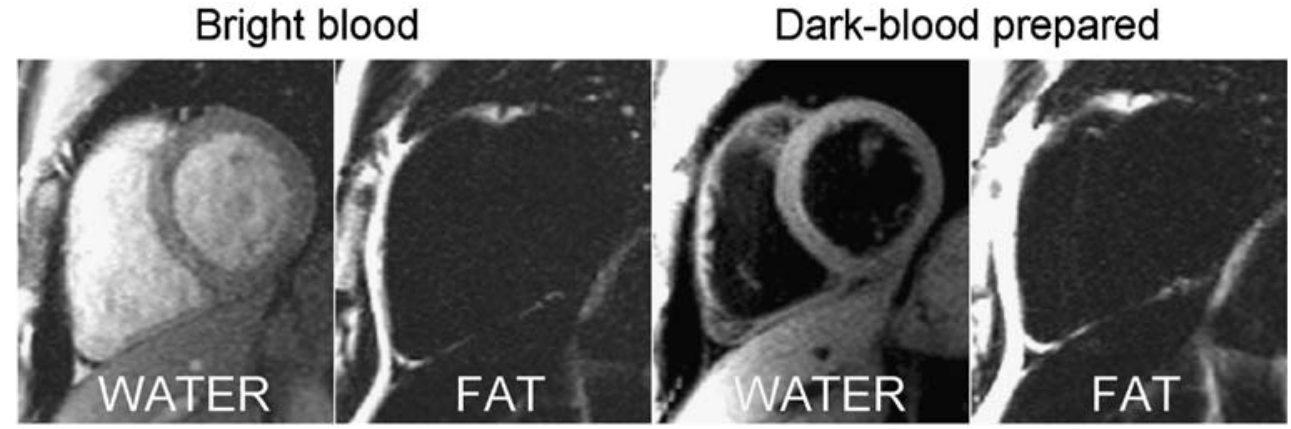

Fig. 1 Dark blood-prepared fat-water-separated images (right) provide better delineation of thin-walled right ventricular myocardium compared with bright blood images (left)

surement of intracellular lipid content within cardiomyocytes. Excessive lipid accumulation within the myocardium is directly cardiotoxic and causes left ventricular remodeling and dilated cardiomyopathy [20]. Singlevoxel magnetic resonance spectroscopy techniques have been used to quantify intracellular lipid levels [21-23] and may discriminate between triglycerides in adipocytes and droplets in the cardiomyocytes based on a small chemical shift. The role of cardiac steatosis in heart disease is being studied in patients with metabolic disorders, such as type II diabetes mellitus [24-26], with ever increasing importance due to the obesity epidemic. The potential for using fat-water-separated imaging techniques for measuring the low-concentration intracellular lipids is being explored [27].

Conventional approaches to fat and water discrimination based on fat suppression are commonly used to characterize masses, but have a reduced ability to characterize myocardial fatty infiltration due to the poor contrast of microscopic fat and partial-volume effects. Multi-echo Dixon-like methods [28, 29•, $30-34 \cdot, 35 \cdot 0]$ for fat and water separation provide a sensitive means of detecting small concentrations of fat with improved contrast. The multi-echo approach to water and fat separation has a number of advantages over chemical-shift fat suppression: 1) fat has positive contrast; 2) water and fat images can be acquired in a single acquisition avoiding spatial misregistration; 3) the method is compatible with pre-contrast and lateenhancement imaging; 4) it is less susceptible to partialvolume effects and 5) robust in the presence of background field variation; and 6) it eliminates chemical-shift artifact.

\section{Fat-Water-Separated Imaging Methods}

There are a number of approaches for imaging fat and characterizing tissue content [36], including 1) use of chemical shift saturation to suppress fat;2) use of T1weighted imaging to detect T1 shortening of fat, which may be observed as bright signal intensity; and 3) use of multiecho methods to reconstruct water- and fat-separated images based on the difference in resonance frequencies between water and fat. Multi-echo methods that jointly estimate the fieldmap, water, and fat images produce excellent discrimination between water and fat. The fat-separated image provides positive contrast (containing only signal from lipids), which improves the diagnostic confidence over conventional chemical shift fat suppression. Limitations of chemical shift fat suppression and T1 measurements for cardiac imaging application are previously described $[11 \bullet \bullet]$.

Dixon's original method for water and fat separation [28] acquires two images with different echo times chosen such that the water and fat are in-phase and opposed-phase, respectively, and may be combined to obtain separate water and fat images. This simple method assumes that the water is exactly on resonance, which limits the performance of water and fat separation in the presence of B0-field inhomogeneity. Large, rapid field variation frequently occurs in the chest due to the geometry of the tissue-air interface. Recent methods $[29 \bullet, 30,32,35 \bullet \cdot]$ for multi-echo Dixon water and fat separation jointly estimate the fieldmap, water, and fat images. These methods apply spatial smoothness constraints
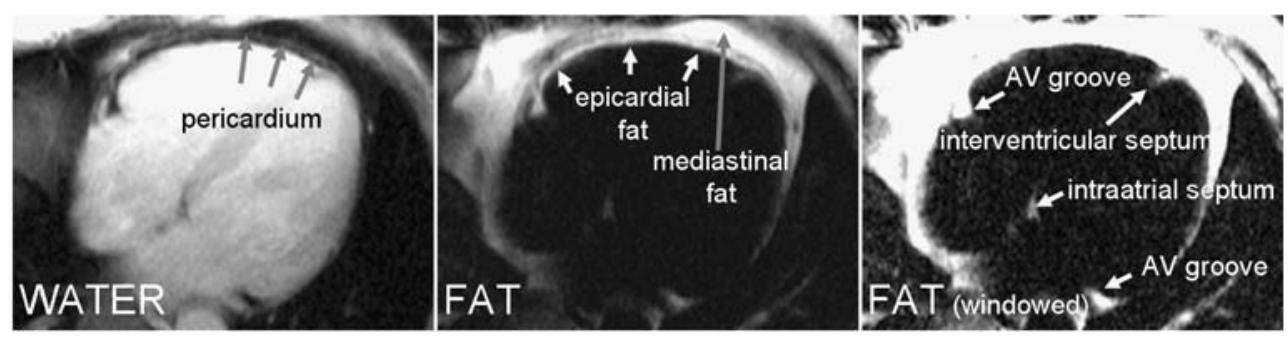

Fig. 2 Water and fat-separated images showing common distribution of fat in and around the heart for a normal subject. The pericardium is seen in the water image (left); the fat image (right) is the same as the middle image, with window-level set to display intraatrial fat and interventricular apical fat with lower signal intensity. AV-atrioventricular 

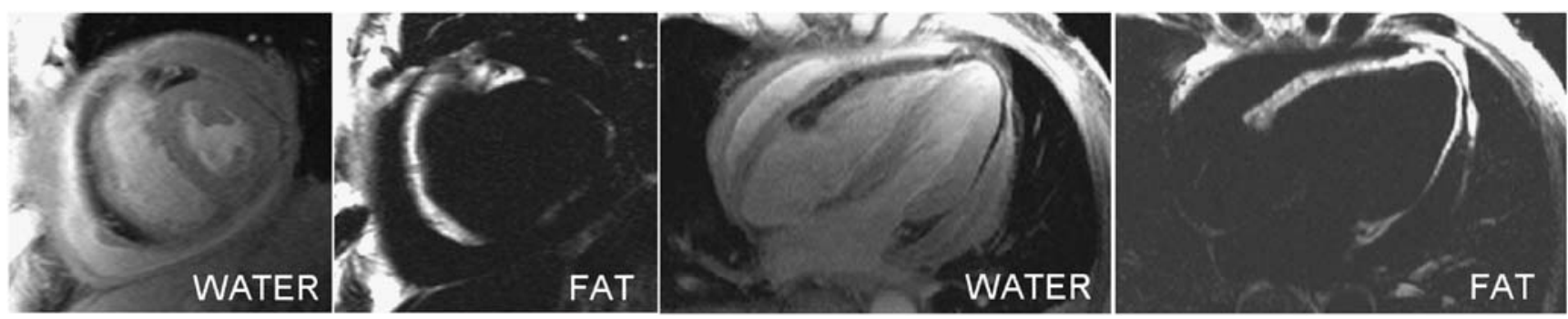

Fig. 3 Fat-water-separated images for a patient with pericardial disease with pericardial effusion and thickened pericardial membrane

in the process of fieldmap estimation $[31,35 \cdot 0]$ and have proven to be robust for cardiac applications $[11 \bullet \bullet, 19 \bullet \bullet]$. These methods may also be used with arbitrary echo times, which allows for more flexible protocols.

A number of methods have been proposed to solve this nonlinear estimation problem. The approach referred to as iterative decomposition with echo asymmetry and leastsquares (IDEAL) [29•, 30] consists of repeated linearizations of the original nonlinear problem, alternatively estimating the water and fat signals and the field map. A number of refinements have been proposed to incorporate more complex signal models to account for $\mathrm{T} 2 *$ relaxation and the multi-peak nature of the fat spectrum [34•]. The improved models provide more reliable fat classification and more accurate quantification of fat fraction. A recently developed joint estimation method for fat-water separation based on a penalized maximum likelihood formulation may be efficiently implemented using variable projection (VARPRO) and graph cuts optimization [35••]. This approach has been applied to cardiac fat-water-separated imaging and has been shown to perform robustly in the presence of large B0field inhomogeneities and at low signal-to-noise ratio (SNR).

\section{Acquisition Protocols}

Multi-echo fat-water-separated imaging has been implemented with a variety of sequences and protocols for both two-dimensional and three-dimensional acquisitions. Sequences include spoiled gradient recalled echo, turbo spin-echo, and steady-state free precession (SSFP), and in specific protocols may include magnetization preparations such as double inversion recovery (DIR) for dark blood contrast and inversion recovery (IR) for late enhancement.

Cardiac imaging using multi-echo sequences is typically performed using an ECG-triggered, segmented acquisition. Breath-held, cine imaging produces fat-water-separated images at a number of cardiac phases. Using cine imaging with continuous radiofrequency drives the magnetization to steady state, and depending on the readout flip angle, has T1-weighted contrast, which helps distinguish the myocardial from adjacent blood pool. However, T1-weighted contrast, if uncorrected, may also lead to overestimation of fat [37•]. While cine imaging is useful for visualizing intramyocardial fat and for characterizing suspected masses, continuous imaging is not compatible with magnetization preparations such as dark-blood or IR. Single cardiac phase acquisitions, typically in mid-diastole, may be used in situations in which magnetization preparation is desired. Single phase imaging acquires data on the approach to steady state, and thus has a somewhat higher SNR and reduced T1weighting for a given readout flip angle, as compared with cine imaging. The reduced T1-weighting improves fat fraction estimates. The reduction in blood pool contrast may be mitigated by using magnetization preparation.

Fat-water imaging may be performed with and without a dark blood preparation, depending on the desired blood pool contrast (Fig. 1). Dark blood DIR-prepared fat-waterseparated imaging provides improved delineation of the myocardium. The thin right ventricular (RV) myocardium is difficult to discern in the bright blood images due to lack of sufficient contrast between RV myocardium and blood. However, the RV free wall is well depicted in the dark blood-prepared images. In this example, the measured T1weighted contrast of between myocardium and blood (water image septal region) was 1:1.5, whereas for the dark blood protocol the contrast was greater than $4: 1$. This improves the ability to discern fatty infiltration of the thinwalled RV myocardium.

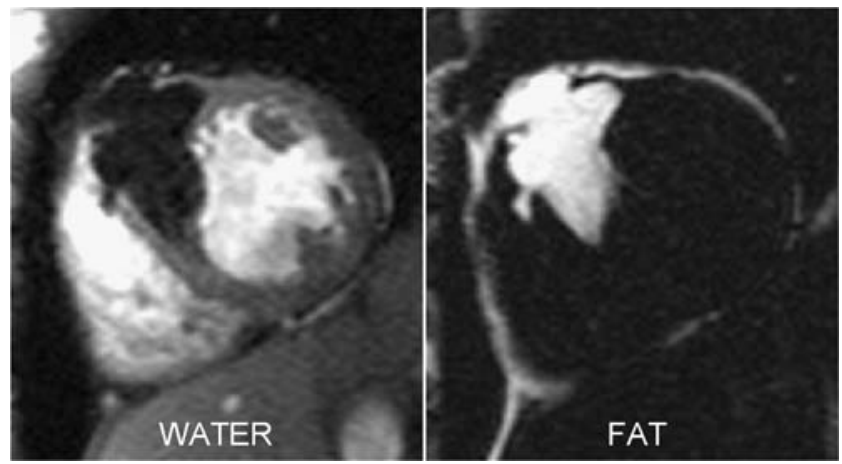

Fig. 4 Pre-contrast images for a patient with a large anteroseptal lipoma acquired in a single breath-hold using a multi-echo fat-waterseparated imaging approach provide excellent contrast between water (left) and fat (right) 


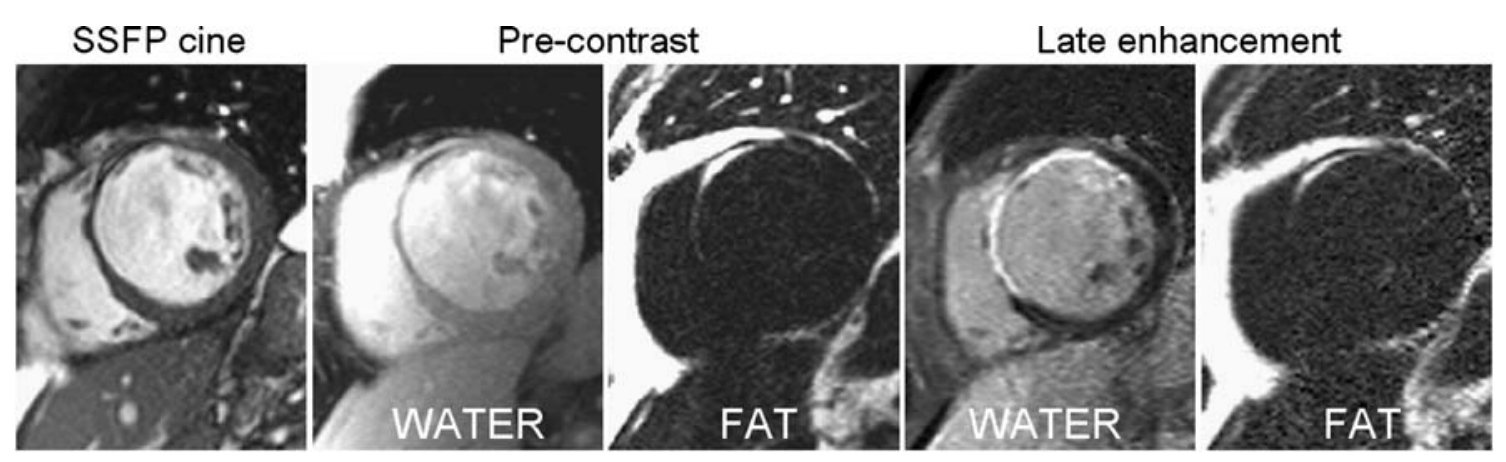

Fig. 5 Patient with chronic myocardial infarction in anteroseptal region showing slight elevated signal intensity in cine steady-state free precession (SSFP) image, fatty infiltration on pre-contrast fat-

Fat-water-separated imaging may be combined with phase-sensitive inversion recovery (PSIR) late enhancement to provide positive correlation between fibrosis and fat, which both appear bright post-contrast. The water and fat images are spatially registered since they are reconstructed from the same multi-echo dataset. Furthermore, chemical shift artifacts may be eliminated in reconstruction.

Multi-echo acquisitions are typically performed using an echo-train readout for improved efficiency. Interleaving with two or more shots may be used for reduced echospacing, particularly at higher field strength to reduce potential ambiguities due to higher chemical shift [38]. Echo-train readout using monopolar readout with gradient flyback is slightly less efficient than bipolar [33] but avoids potential artifacts. The individual echo time images may be noisy as a result of large bandwidth, but the fat and waterseparated images have improved SNR due to the effective signal averaging inherent in the reconstruction. The effective number of signals averaged approaches the number of echoes when the echo spacing is chosen judiciously [39•].

Examples of fat-water-separated images are presented for various clinical cases drawn from our site. Typical parameters for these examples are $1.5 \mathrm{~T}$, single echo train acquisition using monopolar readout with gradient flyback, number of echoes typically 4 , bandwidth approximately water-separated images, and fibrofatty infiltration on phasesensitive inversion recovery late enhancement fat-water-separated images

$1,000 \mathrm{~Hz} /$ pixel, and echo spacing on order of 2.2 to $2.5 \mathrm{~ms}$ with TR approximately 10 to $11 \mathrm{~ms}$. Dark blood or IR preparations were used in specific protocols.

\section{Fat Surrounding and Infiltrating the Heart}

The heart is frequently surrounded by fat, both subepicardial fat within the pericardium, as well as mediastinal fat outside the pericardium. A large amount of mediastinal/pericardial fat encasing the heart has been correlated with increased incidence of coronary artery disease [40]. Fatty infiltration is associated with various cardiomyopathies, as discussed later. Intramyocardial fatty infiltration also appears in normal myocardium [1, 3, 7, 13, 41], with greater prevalence reported in the RV myocardium than in the left ventricle $[7,16]$. In normal hearts, fat is commonly seen in the insertion of inferior right ventricle [7] and in the atrioventricular groove [2]. It is also not uncommon to find adipose tissue in the intraatrial septum (lipomatous hypertrophy) [42]. These common findings are illustrated in Fig. 2 for a normal subject without heart disease. An increased amount of fatty infiltration is also reported in subjects with a large amount of epicardial fat [14], as the excess adipose tissue infiltrates the RV myocardium and atrial septum (lipomatous hypertrophy). Initial findings suggest that there is signifi-
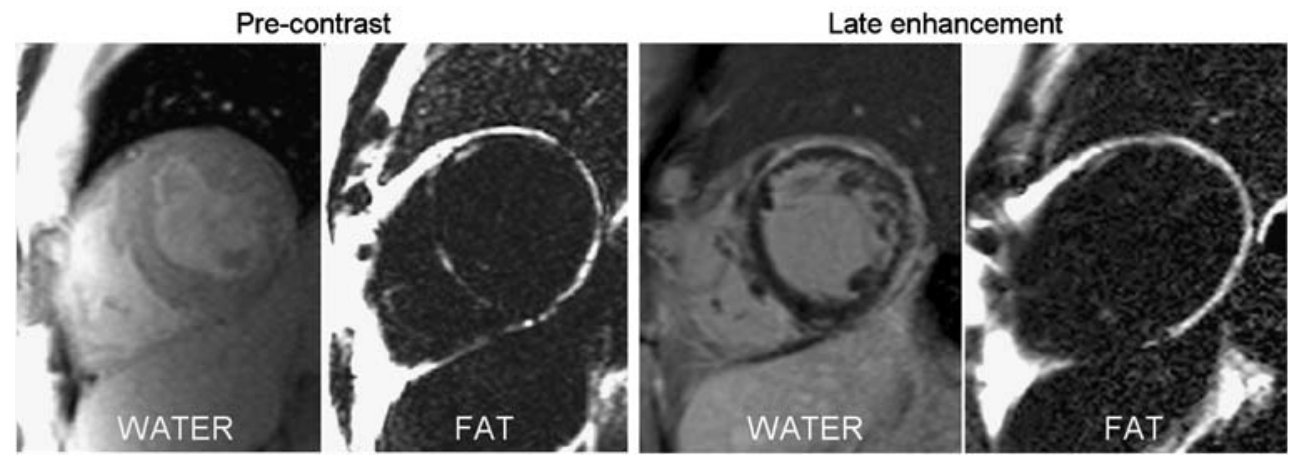

Fig. 6 Fibrofatty infiltration in a patient with nonischemic cardiomyopathy imaged using fat-water-separated imaging pre-contrast (left) and with late enhancement (right) 

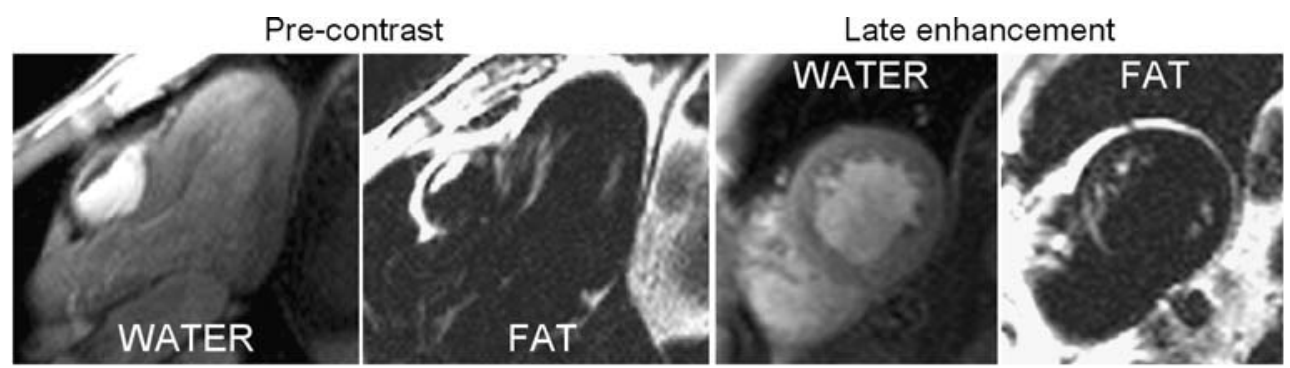

Fig. 7 Fatty infiltration in a patient with Proteus syndrome and with a nonischemic cardiomyopathy shows endocardial fat infiltration in precontrast fat-water-separated images

cance to distinguishing between endocardial versus epicardial infiltration by adipocytes $[1,7,13]$. The border between the epicardial fat and the myocardium is subject to partial volume effects, which places a higher demand for improved spatial resolution to make this distinction clear.

Fat-water separation is useful in anatomical imaging since it provides excellent contrast between adjacent fat and water structures, such as myocardium, epicardial fat, pericardium, and pericardial fat. It also eliminates artifacts such as chemical shift, as discussed later. Figure 3, fat-water-separated images for a patient with pericardial disease, shows a thickened pericardium and discriminates fat structures.

Fat-water imaging has potential for vessel wall imaging. Fat-water-separated imaging of coronary arteries has been proposed as an alternative to using conventional chemical shift fat suppression [38] to provide more uniform contrast. The coronary arteries are typically surrounded by fat and may be visualized with good contrast in the individual water and fat images.

\section{Mass Characterization}

The ability of MRI to discriminate between water and fat is important in tissue characterization. Patients with cardiac masses are frequently referred for MRI to distinguish between thrombosis, cancerous tumors, and fatty lipomas, and to define their detailed morphology. Fat-water-separated imaging provides excellent contrast and is a useful tool in conjunction with other measures, such as $\mathrm{T} 1$ and T2weighted, contrast enhanced perfusion, and late enhancement.

Conventional chemical shift fat suppression using DIR turbo or fast-spin echo readout is a standard method for characterizing fat. This method is typically performed with and without fat suppression in two separate breath-holds. Chemical shift fat suppression has limited performance due to variation of the off-resonance shift caused by magnetic field inhomogeneities across the field-of-view. Fat-waterseparated imaging (Fig. 4) may be performed in a single breath-hold acquisition and provides uniformly good contrast across the field-of-view.

\section{Ischemic Cardiomyopathy}

Conventional viability imaging using late enhancement does not distinguish fibrosis from fibrofatty infiltration [5]. Fat-water-separated late enhancement imaging has recently been introduced $[11 \bullet \bullet, 12]$ and may be used be used to directly image fat in chronic MI. A number of cardiac CT studies have also reported the presence of fat associated with chronic MI [4-6]. The measurement of fibrofatty infiltration in chronic MI by imaging studies is relatively new. As a result, the prevalence and factors that influence fatty infiltration in chronic MI, as well as the clinical implications, are largely unknown.

An example of fat-water-separated imaging of chronic MI in the anteroseptal region is shown in Fig. 5. Elevated
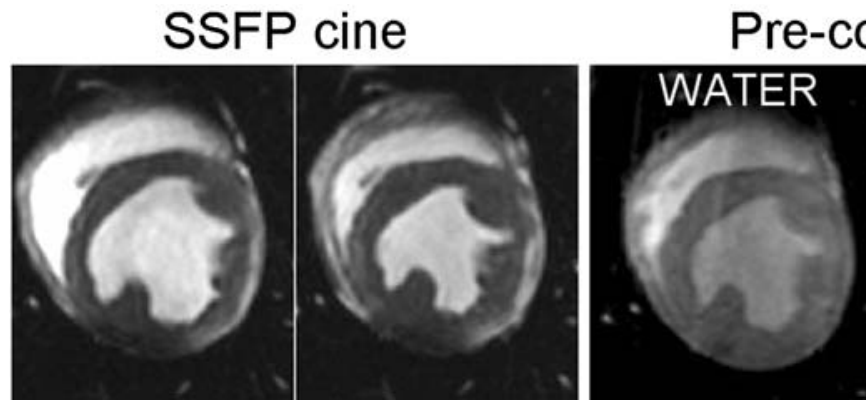

ontrast

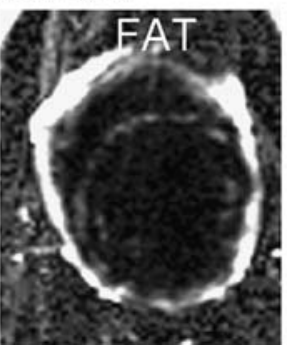

Late enhancement

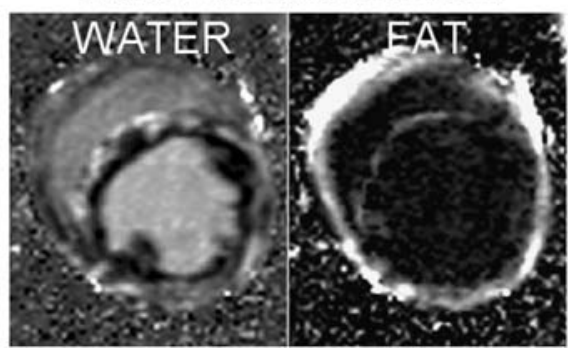

Fig. 8 Duchenne muscular dystrophy in a canine model with dilated cardiomyopathy showing diastolic and systolic phases of steady-state free precession (SSFP) cine images; intramyocardial fat is clearly evident in pre-contrast fat-water-separated images; fibrofatty infiltration is clearly evident in phase-sensitive inversion recovery late enhancement fat-water-separated images 


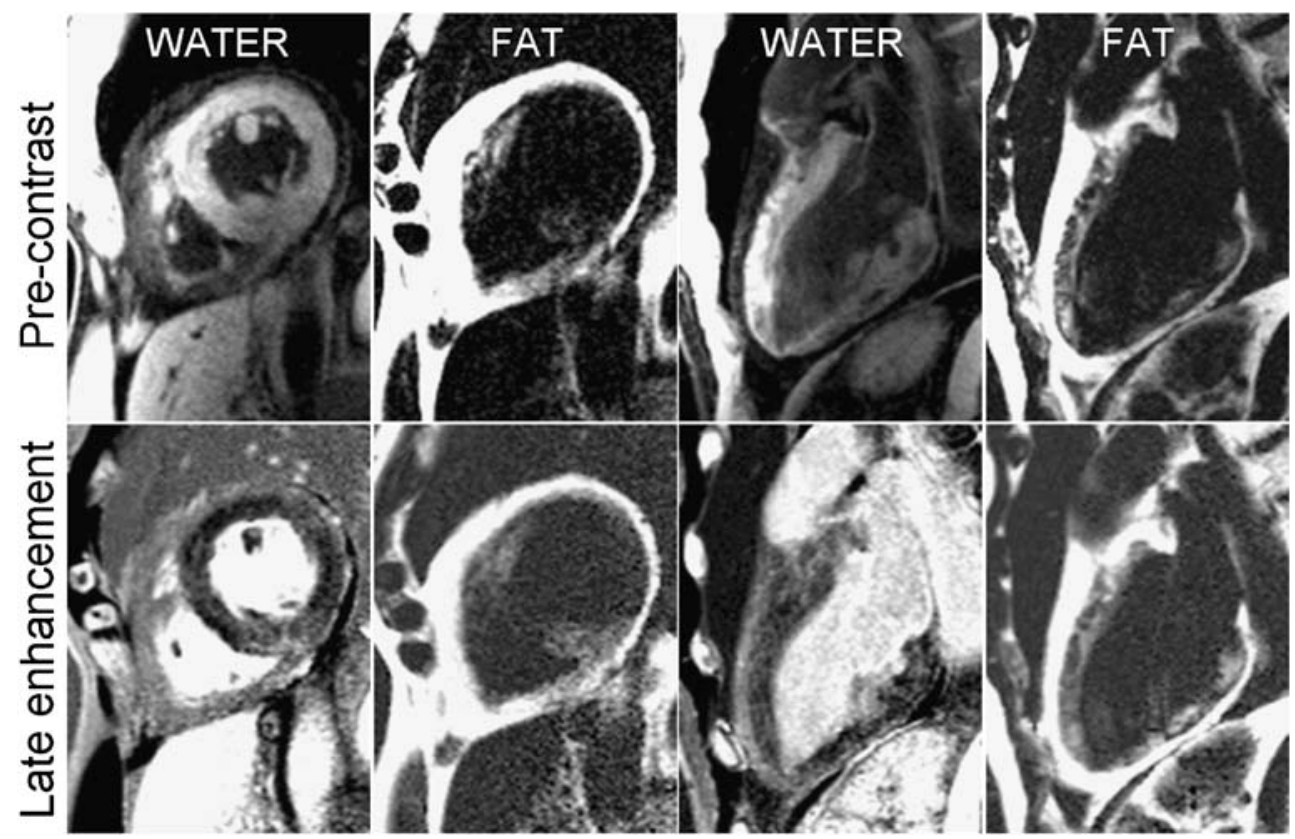

Fig. 9 Patient with lipophilic disorder with extensive fatty infiltration of both left ventricular and right ventricular myocardium seen in dark blood-prepared pre-contrast (top row) and late enhancement (bottom row) images

signal intensity is evident in the corresponding SSFP cine image for a region of the MI. This is due to the lower T1 of fat since SSFP images have contrast proportional to $\sqrt{ }(\mathrm{T} 2 / \mathrm{T} 1)$. An elevated T2 may also appear bright, which may arise in cases of acute MI or myocarditis and would be difficult to distinguish. Fatty infiltration is clearly seen in both precontrast fat-water-separated images and PSIR late enhancement fat-water-separated images.

\section{Nonischemic Cardiomyopathy}

Fibrofatty infiltration of the myocardium is associated with a number of nonischemic cardiomyopathies. It is of specific interest to distinguish between isolated fat regions and regions of fibrous fat, and to determine the degree and distribution of fat and fibrous fat regions. Several studies [1, 2, 13, 15] have assessed the presence of intramyocardial fat though histo- logical studies from endocardial biopsy and/or autopsies. Imaging has the potential for larger-scale studies of prevalence of intramyocardial fat and correlation with cardiomyopathy. Imaging must classify tissue as 1) isolated fatty infiltration versus 2) fibrofatty infiltration, and distinguish between 1) endocardial versus 2) epicardial distribution of infiltration. It may also be important to assess the fat fraction (ie, percentage of fat in a voxel). High spatial resolution is important to minimize partial volume effects.

An example of fibrofatty infiltration in a patient with nonischemic cardiomyopathy is shown in Fig. 6. Fatty infiltration of the myocardium is seen in both pre-contrast and late-enhancement fat-water-separated images in the interventricular septum and anteroseptal regions. The fibrofatty tissue is subepicardial in distribution in this case. A second example (Fig. 7) of fatty infiltration in a patient with nonischemic cardiomyopathy and Proteus syndrome

\section{Conventional}

late enhancement

\section{Pre-contrast}
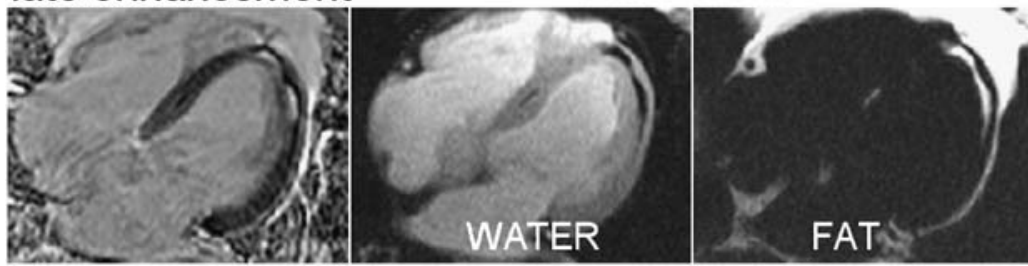

\section{Late enhancement}

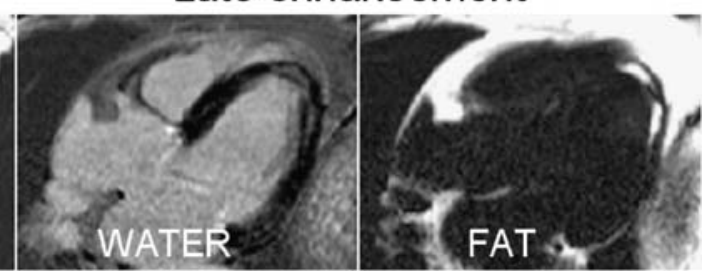

Fig. 10 Example of a patient referred for suspected coronary artery disease who exhibited a hyperintense signal in the apical region of the left ventricular myocardium on conventional late enhancement that is actually due to intramyocardial fat. Intramyocardial fat appears as (bright) chronic myocardial infarction on the conven- tional late-enhancement image; however, pre-contrast water and fat, as well as water and fat-separated phase-sensitive inversion recovery late-enhancement images, clearly indicate intramyocardial fat in apical and intraventricular regions 
shows a distribution of endocardial fat infiltration. Patients with Proteus syndrome have a reported increased incidence of fatty infiltration of skeletal muscle [43].

Progressive cardiomyopathy leading to congestive heart failure is a common form of death in Duchenne muscular dystrophy (DMD). The use of late enhancement in cardiac magnetic resonance (CMR) imaging for assessing fibrosis in DMD and correlating with left ventricular (LV) function has recently been reported [44, 45]. Fatty replacement in fibrotic tissue associated with cardiomyopathy in cases of DMD is readily observed in histology [46]. The significance and progression of fatty replacement in DMD is currently unknown, although inducibility of arrhythmias has been reported to correlate with fatty infiltration [18]. Fat-water imaging was used in a pilot study to investigate fibrofatty infiltration in a canine model with DMD [47]. Fibrofatty infiltration was observed circumferentially in the left ventricle, emanating from the epicardial surface (Fig. 8). Note that the fat images pre- and post-contrast are similar with respect to extent and location of intramyocardial fat. The fibrotic tissue observed in the late-enhancement water image coincides with the region of fatty infiltration but has greater transmural extent. Cine functional images (left) acquired with SSFP show slight hyperenhancement due to the low T1 of fat. The DMD progression in this case led to dilated cardiomyopathy with a low ejection fraction on order of $25 \%$.

Fibrofatty infiltration in arrhythmogenic right ventricular dysplasia/cardiomyopathy (ARVD/C) has been established through both autopsy/biopsy as well as imaging studies [1, $10,13,17]$, yet the ARVD Taskforce guidelines for diagnosis by CMR do not include the presence of fat as criteria [48]. This is due in part to the subjectivity of interpreting fat based on conventional chemical fat suppression imaging $[9,48]$. This may also be due to the relatively poor contrast of conventional methods, or due to the limited correlation with late enhancement acquired on separate breath-holds. Fat-water-separated imaging offers a new tool with potential for improved reliability [11・•] in CMR diagnosis of fibrofatty infiltration.

Figure 9 shows an example of a patient with a lipophilic disorder with extensive fatty infiltration that is nonischemic in nature. This patient did not meet the Taskforce criteria for ARVD. Intramyocardial fat appears diffusely throughout both LV and RV myocardium, and there is some atypical late enhancement indicating possible diffuse fibrosis.

\section{Artifact Reduction}

Fat-water-separated imaging may ensure correct recognition of fat and avoid erroneous tissue classification. In one instance, in SSFP imaging commonly used for cardiac cine, fat may appear hyperintense and may be confused with elevated T2 associated with edema, seen for example in Figs. 5 and 8. With late enhancement imaging, it may be difficult to discriminate between fibrosis and intramyocardial fat since both have low T1 and appear bright (Fig. 10). In this case it may be difficult to distinguish fat from MI or other fibrotic scar tissue. Late enhancement imaging is widely used for viability imaging of MI and is increasingly used more generally to characterize atypical fibrosis associated with nonischemic cardiomyopathies [49]. Using fat-water-separated late-enhancement imaging it is possible to distinguish the fibrosis from fat $[11 \bullet \bullet]$.

A benefit of the multi-echo fat-water-separated imaging method is the mitigation of the chemical shift artifact. Conventional late enhancement imaging [50] using $140 \mathrm{~Hz} /$ pixel has significant chemical shift artifact in which the fat is displaced relative to the water in the readout direction by approximately 1.5 pixels. In this case, the epicardial fat may be displaced by as much as $30 \%$ of the diastolic wall thickness. In contrast, the multi-echo fat-water-separated imaging approach, which uses a much larger bandwidth, has a sub-pixel shift (typically 0.2 pixel) and may be completely eliminated in the reconstruction by applying the known sub-pixel shift to the fat image.

\section{Conclusions}

Fat and water-separated imaging provides a sensitive means of detecting intramyocardial fat with positive signal contrast, thereby achieving a high degree of confidence, whereas conventional fat suppression is often difficult to interpret due to fluctuations in the water signal. The water and fat separation method may be combined with lateenhancement imaging to provide a positive correlation between fibrosis and fat, both acquired simultaneously, and therefore, spatially registered. This technique may be used to assess the prognostic value of the presence and amount of myocardial fat and fibrofatty infiltration. Fat and waterseparated imaging has the potential for quantitative measurement of fat fraction in the heart, which could have application to metabolic disorders. Furthermore, fat and water-separated imaging is useful in resolving artifacts that may arise in other protocols caused by the presence of fat.

Acknowledgment This research was supported by the Intramural Research Program of the National Institutes of Health, National Heart, Lung, and Blood Institute, and a Cooperative Research and Development Agreement between the National Heart, Lung, and Blood Institute and Siemens Medical Solutions.

Disclosure No potential conflicts of interest relevant to this article were reported.

Open Access This article is distributed under the terms of the Creative Commons Attribution Noncommercial License which permits any noncommercial use, distribution, and reproduction in any medium, provided the original author(s) and source are credited. 


\section{References}

Papers of particular interest, published recently, have been highlighted as follows:

- Of importance

•- Of major importance

1. Burke AP, Farb A, Tashko G, Virmani R: Arrhythmogenic right ventricular cardiomyopathy and fatty replacement of the right ventricular myocardium: are they different diseases? Circulation 1998, 97:1571-1580

2. Caruso G, Frassanito F, Serio G, Pennella A: Is adipose tissue a normal component of the myocardium? Eur Heart J 1989, 10 (Suppl D):89-91.

3. Fontaine G, Fontaliran F, Zenati $\mathrm{O}$, et al.: Fat in the heart. A feature unique to the human species? Observational reflections on an unsolved problem. Acta Cardiol 1999, 54:189-194.

4. Winer-Muram HT, Tann M, Aisen AM, et al.: Computed tomography demonstration of lipomatous metaplasia of the left ventricle following myocardial infarction. J Comput Assist Tomogr 2004, 28:455-458.

5. Wu YW, Tadamura E, Yamamuro M, et al.: Identification of lipomatous metaplasia in old infarcted myocardium by cardiovascular magnetic resonance and computed tomography. Int J Cardiol 2007, 115:e15-e16.

6. Robles P, Sonlleva A: Myocardial calcification and subendocardial fatty replacement of the left ventricle following myocardial infarction. Int J Cardiovasc Imaging 2007, 23:667-670.

7. Raney AR, Saremi F, Kenchaiah S, et al.: Multidetector computed tomography shows intramyocardial fat deposition. J Cardiovasc Comput Tomogr 2008, 2:152-163.

8. Tandri H, Saranathan M, Rodriguez ER, et al.: Noninvasive detection of myocardial fibrosis in arrhythmogenic right ventricular cardiomyopathy using delayed-enhancement magnetic resonance imaging. J Am Coll Cardiol 2005, 45:98-103.

9. Tandri H, Castillo E, Ferrari VA, et al.: Magnetic resonance imaging of arrhythmogenic right ventricular dysplasia: sensitivity, specificity, and observer variability of fat detection versus functional analysis of the right ventricle. J Am Coll Cardiol 2006, 48:2277-2284.

10. Sen-Chowdhry S, Prasad SK, Syrris P, et al.: Cardiovascular magnetic resonance in arrhythmogenic right ventricular cardiomyopathy revisited: comparison with task force criteria and genotype. J Am Coll Cardiol 2006, 48:2132-2140.

11. • Kellman P, Hernando D, Shah S, et al.: Multi-echo Dixon fat and water separation method for detecting fibro-fatty infiltration in the myocardium. Magn Reson Med 2009, 61:215-221. This article describes cardiac applications for fat-water-separated imaging, methods for fat and water-separated late enhancement imaging, and comparison with conventional chemical shift fat suppression.

12. Goldfarb JW: Fat-water separated delayed hyperenhanced myocardial infarct imaging. Magn Reson Med 2008, 60:503-509.

13. Fontaliran F, Fontaine G, Fillette F, et al.: Nosologic frontiers of arrhythmogenic dysplasia. Quantitative variations of normal adipose tissue of the right heart ventricle. Arch Mal Coeur Vaiss 1991, 84:33-38.

14. Shirani J, Berezowski K, Roberts WC: Quantitative measurement of normal and excessive (cor adiposum) subepicardial adipose tissue, its clinical significance, and its effect on electrocardiographic QRS voltage. Am J Cardiol 1995, 76:414-418.

15. Baroldi G, Silver MD, De Maria R, et al.: Lipomatous metaplasia in left ventricular scar. Can J Cardiol 1997, 13:65-71.

16. $\mathrm{Su} \mathrm{L}$, Siegel JE, Fishbein MC: Adipose tissue in myocardial infarction. Cardiovasc Pathol 2004, 13:98-102.
17. Molinari G, Sardanelli F, Zandrino F, et al.: Adipose replacement and wall motion abnormalities in right ventricle arrhythmias: evaluation by MR imaging. Retrospective evaluation on 124 patients. Int J Card Imaging 2000, 16:105-115. Erratum in Int J Card Imaging 2000, 16:485.

18. Vignaux $\mathrm{O}$, Lazarus A, Varin $\mathrm{J}$, et al.: Right ventricular MR abnormalities in myotonic dystrophy and relationship with intracardiac electrophysiologic test findings: initial results. Radiology 2002, 224:231-235.

19. •• Reeder SB, Markl M, Yu H, et al.: Cardiac CINE imaging with IDEAL water-fat separation and steady-state free precession. J Magn Reson Imaging 2005, 22:44-52. This article describes cardiac applications for fat-water-separated imaging.

20. McGavock JM, Victor RG, Unger RH, Szczepaniak LS: Adiposity of the heart, revisited. Ann Intern Med 2006, 144:517-524.

21. den Hollander JA, Evanochko WT, Pohost GM: Observation of cardiac lipids in humans by localized $1 \mathrm{H}$ magnetic resonance spectroscopic imaging. Magn Reson Med 1994, 32:175-180.

22. Szczepaniak LS, Dobbins RL, Metzger GJ, et al.: Myocardial triglycerides and systolic function in humans: in vivo evaluation by localized proton spectroscopy and cardiac imaging. Magn Reson Med 2003, 49:417-423.

23. van der Meer RW, Doornbos J, Kozerke S, et al.: Metabolic imaging of myocardial triglyceride content: reproducibility of $1 \mathrm{H}$ MR spectroscopy with respiratory navigator gating in volunteers. Radiology 2007, 245:251-257.

24. McGavock JM, Lingvay I, Zib I, et al.: Cardiac steatosis in diabetes mellitus: a $1 \mathrm{H}$-magnetic resonance spectroscopy study. Circulation 2007, 116:1110-1112.

25. Szczepaniak LS, Victor RG, Orci L, Unger RH: Forgotten but not gone: the rediscovery of fatty heart, the most common unrecognized disease in America. Circ Res 2007, 101:759-767.

26. Rijzewijk LJ, van der Meer RW, Smit JW, et al.: Myocardial steatosis is an independent predictor of diastolic dysfunction in type 2 diabetes mellitus. J Am Coll Cardiol 2008, 52:17931799.

27. Liu CY, Redheuil A, Ouwerkerk R, et al.: Myocardial fat quantification using 2D Dixon MRI: feasibility study. J Cardiovasc Magn Reson 2009, 11(Suppl 1):107.

28. Dixon W: Simple proton spectroscopic imaging. Radiology 1984 , 153:189-194.

29. • Reeder SB, Wen Z, Yu H, et al.: Multicoil Dixon chemical species separation with an iterative least squares estimation method. Magn Reson Med 2004, 51:35-45. This article details initial development of the IDEAL algorithm for fat-water-separated imaging.

30. Reeder SB, Pineda AR, Wen Z, et al.: Iterative decomposition of water and fat with echo asymmetry and least-squares estimation (IDEAL): application with fast-spin echo imaging. Magn Reson Med 2005, 54:636-644.

31. Yu H, Reeder SB, Shimakawa A, et al.: Field map estimation with a region growing scheme for iterative 3-point water-fat decomposition. Magn Reson Med 2005, 54:1032-1039.

32. Hernando D, Haldar JP, Sutton BP, et al.: Joint estimation of water/fat images and field inhomogeneity map. Magn Res Med 2008, 59:571-580.

33. Lu W, Yu H, Shimakawa A, et al.: Water-fat separation with bipolar multiecho sequences. Magn Reson Med 2008, 60:198209.

34. • Yu H, Shimakawa A, McKenzie CA, et al.: Multiecho water fat separation and simultaneous R2* estimation with multifrequency fat spectrum modeling. Magn Reson Med 2008, 60:1122-1134. This article describes the importance of improved fat models on performance of fat and water separation.

35. • Hernando D, Kellman P, Haldar JP, Liang ZP: Robust water/fat separation in the presence of large field inhomogeneities using a 
graph cut algorithm. Magn Reson Med 2010, 63:79-90. This article presents a robust algorithm for fat-water-separated imaging in the heart.

36. • Bley TA, Wieben O, François CJ, et al.: Fat and water magnetic resonance imaging. J Magn Reson Imaging 2010, 31:4-18. This is a review of methods for fat and water imaging.

37. - Liu CY, McKenzie CA, Yu H, et al.: Fat quantification with IDEAL gradient echo imaging: correction of bias from $\mathrm{T}(1)$ and noise. Magn Reson Med 2007, 58:354-364. This article describes important considerations for fat quantification.

38. Shah S, Bi X, Hernando D, et al.: Coronary MRA at 3T using 3d multi-interleaved multi-echo acquisition with varpro fat-water separation. J Cardiovasc Magn Reson 2010, 12(Suppl 1):P42

39. • Pineda AR, Reeder SB, Wen Z, Pelc NJ: Cramér-Rao bounds for three-point decomposition of water and fat. Magn Reson Med 2005, 54:625-635. This article presents guidelines for selection of echo times and formulation of effective averaging.

40. Taguchi R, Takasu J, Itani Y, et al.: Pericardial fat accumulation in men as a risk factor for coronary artery disease. Atherosclerosis 2001, 157:203-209.

41. Macedo R, Prakasa K, Tichnell C, et al.: Marked lipomatous infiltration of the right ventricle: MRI findings in relation to arrhythmogenic right ventricular dysplasia. AJR Am J Roentgenol 2007, 188:W423-W427.

42. O'Connor S, Recavarren R, Nichols LC, Parwani AV: Lipomatous hypertrophy of the interatrial septum: an overview. Arch Pathol Lab Med 2006, 130:397-399.
43. Jamis-Dow CA, Turner J, Biesecker LG, Choyke PL: Radiologic manifestations of Proteus syndrome. Radiographics 2004, 24:1051-1068.

44. Silva MC, Meira ZM, Gurgel Giannetti J, et al.: Myocardial delayed enhancement by magnetic resonance imaging in patients with muscular dystrophy. J Am Coll Cardiol 2007, 49:1874-1879.

45. Puchalski MD, Williams RV, Askovich B, et al.: Late gadolinium enhancement: precursor to cardiomyopathy in Duchenne muscular dystrophy? Int J Cardiovasc Imaging 2009, 25:57-63.

46. Frankel KA, Rosser RJ: The pathology of the heart in progressive muscular dystrophy: epimyocardial fibrosis. Hum Pathol 1976, 7:375-386.

47. Kellman P, Hernando D, Shah S, et al.: Myocardial fibro-fatty infiltration in Duchenne muscular dystrophy canine model detected using multi-echo Dixon method of water and fat separation imaging. Proc Intl Soc Mag Reson Med 2009, 17:3762.

48. Bluemke DA, Krupinski EA, Ovitt T, et al.: MR imaging of arrhythmogenic right ventricular cardiomyopathy: morphologic findings and interobserver reliability. Cardiology 2003, 99:153162.

49. Hunold P, Schlosser T, Vogt FM, et al.: Myocardial late enhancement in contrast-enhanced cardiac MRI: distinction between infarction scar and non-infarction-related disease. AJR Am J Roentgenol 2005, 184:1420-1426.

50. Kim RJ, Shah DJ, Judd RM: How we perform delayed enhancement imaging. J Cardiovasc Magn Reson 2003, 5:505514. Erratum in J Cardiovasc Magn Reson 2003, 5:613-615. 